Bond University

Research Repository

\title{
Updating guidance for reporting systematic reviews: development of the PRISMA 2020 statement
}

Page, Matthew J; McKenzie, Joanne E; Bossuyt, Patrick M; Boutron, Isabelle; Hoffmann, Tammy C; Mulrow, Cynthia D; Shamseer, Larissa; Tetzlaff, Jennifer M; Moher, David Published in:

Journal of Clinical Epidemiology

DOI:

10.1016/j.jclinepi.2021.02.003

Licence:

CC BY-NC-ND

Link to output in Bond University research repository.

Recommended citation(APA):

Page, M. J., McKenzie, J. E., Bossuyt, P. M., Boutron, I., Hoffmann, T. C., Mulrow, C. D., Shamseer, L., Tetzlaff, J. M., \& Moher, D. (2021). Updating guidance for reporting systematic reviews: development of the PRISMA 2020 statement. Journal of Clinical Epidemiology, 134, 103-112. https://doi.org/10.1016/j.jclinepi.2021.02.003

\section{General rights}

Copyright and moral rights for the publications made accessible in the public portal are retained by the authors and/or other copyright owners and it is a condition of accessing publications that users recognise and abide by the legal requirements associated with these rights.

For more information, or if you believe that this document breaches copyright, please contact the Bond University research repository coordinator. 


\section{Journal Pre-proof}

Updating guidance for reporting systematic reviews: development of the PRISMA 2020 statement

Matthew J Page, Joanne E McKenzie, Patrick M Bossuyt , Isabelle Boutron, Tammy C Hoffmann, Cynthia D Mulrow, Larissa Shamseer, Jennifer M Tetzlaff, David Moher

PII: S0895-4356(21)00040-8

DOI: https://doi.org/10.1016/j.jclinepi.2021.02.003

Reference: JCE 10417

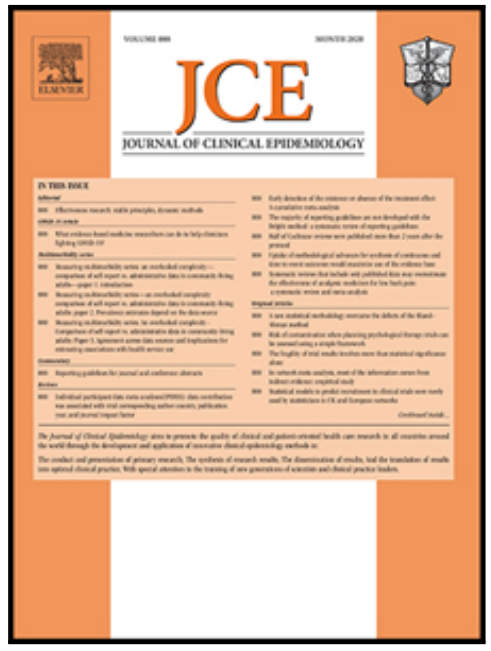

To appear in:

Journal of Clinical Epidemiology

Accepted date: $\quad 3$ February 2021

Please cite this article as: Matthew J Page, Joanne E McKenzie, Patrick M Bossuyt , Isabelle Boutron, Tammy C Hoffmann, Cynthia D Mulrow, Larissa Shamseer, Jennifer M Tetzlaff, David Moher, Updating guidance for reporting systematic reviews: development of the PRISMA 2020 statement, Journal of Clinical Epidemiology (2021), doi: https://doi.org/10.1016/j.jclinepi.2021.02.003

This is a PDF file of an article that has undergone enhancements after acceptance, such as the addition of a cover page and metadata, and formatting for readability, but it is not yet the definitive version of record. This version will undergo additional copyediting, typesetting and review before it is published in its final form, but we are providing this version to give early visibility of the article. Please note that, during the production process, errors may be discovered which could affect the content, and all legal disclaimers that apply to the journal pertain.

(C) 2021 Published by Elsevier Inc. 


\section{What is new?}

\section{Key findings}

- A review of 60 documents providing reporting guidance for systematic reviews revealed that all topics addressed by the PRISMA 2009 statement could be modified.

- Of the 110 respondents to survey about potential modifications to the PRISMA 2009 statement, more than $66 \%$ recommended keeping six of the original checklist items as they were and modifying 15 of them using wording suggested by us.

- Attendees at an in-person meeting supported the revised wording for several PRISMA items but suggested rewording for most to enhance clarity, and further refinements were made over six drafts of the PRISMA 2020 statement.

\section{What this adds to what is known}

- The PRISMA 2020 statement replaces the PRISMA 2009 statement and consists of updated reporting guidance for systematic reviews, reflecting advances in methods to identify, select, appraise and synthesize studies.

\section{What is the implication, what should change now?}

- Developers of new and updated reporting guidelines should disseminate a detailed description of their development methods.

- Authors, editors and peer reviewers should adopt the PRISMA 2020 statement when authoring, editing or reviewing systematic reviews. 
Updating guidance for reporting systematic reviews: development of the PRISMA 2020 statement

Matthew J Page ${ }^{1, *}$, Joanne E McKenzie ${ }^{1, \dagger}$, Patrick M Bossuyt ${ }^{2}$, Isabelle Boutron ${ }^{3}$, Tammy C Hoffmann $^{4}$, Cynthia D Mulrow ${ }^{5}$, Larissa Shamseer ${ }^{6}$, Jennifer M Tetzlaff ${ }^{7}$ David Moher $^{8, \dagger}$

1. School of Public Health and Preventive Medicine, Monash University, Melbourne, Victoria, Australia

2. Department of Clinical Epidemiology, Biostatistics and Bioinformatics, Amsterdam University Medical Centres, University of Amsterdam, Amsterdam, Netherlands

3. Université de Paris, Centre of Epidemiology and Statistics (CRESS), Inserm, F 75004 Paris, France

4. Institute for Evidence-Based Healthcare, Faculty of Health Sciences and Medicine, Bond University, Gold Coast, Australia

5. University of Texas Health Science Center at San Antonio, San Antonio, Texas, United States; Annals of Internal Medicine

6. School of Epidemiology and Public Health, Faculty of Medicine, University of Ottawa, Ottawa, Canada

7. Evidence Partners, Ottawa, Canada

8. Centre for Journalology, Clinical Epidemiology Program, Ottawa Hospital Research Institute, Ottawa, Canada; School of Epidemiology and Public Health, Faculty of Medicine, University of Ottawa, Ottawa, Canada 
*Correspondence to: Dr. Matthew Page, School of Public Health and Preventive Medicine,

Monash University, 553 St Kilda Road, Melbourne, Victoria, 3004, Australia. Telephone: +61 9903 0248. Email address: matthew.page@monash.edu

$\dagger \mathrm{JEM}$ and DM are joint senior authors. 


\begin{abstract}
Objectives: To describe the processes used to update the PRISMA 2009 statement for reporting systematic reviews, present results of a survey conducted to inform the update, summarise decisions made at the PRISMA update meeting, and describe and justify changes made to the guideline.
\end{abstract}

Methods: We reviewed 60 documents with reporting guidance for systematic reviews to generate suggested modifications to the PRISMA 2009 statement. We invited 220 systematic review methodologists and journal editors to complete a survey about the suggested modifications. The results of these projects were discussed at a 21 -member in-person meeting. Following the meeting, we drafted the PRISMA 2020 statement and refined it based on feedback from co-authors and a convenience sample of 15 systematic reviewers.

Results: The review of 60 documents revealed that all topics addressed by the PRISMA 2009 statement could be modified. Of the 110 survey respondents, more than $66 \%$ recommended keeping six of the original checklist items as they were and modifying 15 of them using wording suggested by us. Attendees at the in-person meeting supported the revised wording for several items but suggested rewording for most to enhance clarity, and further refinements were made over six drafts of the guideline.

Conclusions: The PRISMA 2020 statement consists of updated reporting guidance for systematic reviews. We hope that providing this detailed description of the development process will enhance the acceptance and uptake of the guideline and assist those developing and updating future reporting guidelines.

Word count: 4,889 


\section{Introduction}

In 2009, an international group of systematic reviewers, methodologists, clinicians and journal editors disseminated the Preferred Reporting Items for Systematic reviews and MetaAnalyses (PRISMA) statement, a guideline designed to help authors prepare a complete report of their systematic review (1-7). The PRISMA 2009 statement included 27 checklist items representing a minimum set of information to convey in a systematic review report, covering the rationale for the review, databases used to identify studies, results of metaanalyses conducted, and implications of the review findings. Each checklist item was accompanied by an 'explanation and elaboration' providing rationale and additional pedagogical guidance for each item, along with exemplars to demonstrate complete reporting (8-12). Uptake of the guideline has been extensive, as indicated by its citation in tens of thousands of systematic reviews and frequent use as a tool to evaluate the completeness of reporting of published systematic reviews (13). To ensure its currency and relevance, in 2017 we set out to update the PRISMA 2009 statement by incorporating advances in systematic review methodology and terminology occurring in the last decade.

Leaders of the EQUATOR (Enhancing the QUAlity and Transparency Of health Research) Network have produced methodological guidance for developers of new health research reporting guidelines (14). However, no methodological advice for updating a reporting guideline exists, and only a few reporting guidelines have been updated (15-18). Therefore, providing a detailed description of the updating process offers a potential roadmap for others embarking on a similar initiative. Furthermore, documenting the evidence, theoretical considerations and rationale for the reporting recommendations is important for future updates and, in addition, may be of interest to users wondering why particular recommendations were made. In this paper, we describe the steps taken to update the 
PRISMA 2009 statement, summarise the key changes made to the guideline, and provide a rationale for these changes.

\section{Methods}

We established an international core team of individuals with expertise in systematic review and meta-analysis methodology and reporting guideline development, and experience in authoring and editing systematic reviews, to lead the update of PRISMA (MJP, JEM, PMB, IB, TH, CDM, LS and DM). We registered the update with the EQUATOR Network website on 13 December 2017 (19), and our protocol was uploaded to the Open Science Framework repository on 14 February 2018 (20). Deviations from the protocol are noted in supplement 1 in the Appendix. We adapted the EQUATOR Network's guidance for developing health research reporting guidelines (14) and the methods used to update the CONSORT and STARD guidelines (15-18). This involved a 4-step process of (i) reviewing relevant literature to identify possible modifications to the PRISMA 2009 items and possible new items, (ii) conducting a survey of systematic review methodologists and editors to gather feedback on the items, (iii) holding a meeting to discuss items, and (iv) drafting and refining the guidance. Further detail is provided below for each step.

\subsection{Step 1. Review of literature to inform the guideline}

We reviewed several types of literature to identify items that could be modified, added to, or removed from the PRISMA 2009 statement. We revisited four prior evaluations of the reporting of published systematic reviews (described in detail elsewhere $(13,21-23)$ ) and noted any PRISMA 2009 items not reported in at least a third of the reviews sampled, to identify potentially problematic items within the current checklist. We collated items included in other documents providing reporting guidance for systematic reviews and 
mapped them against the items recommended in the PRISMA 2009 statement, to determine what, if anything, was missing from PRISMA 2009 (described fully elsewhere (24)). In brief, we reviewed 60 documents, consisting of the PRISMA 2009 statement and its 10 extensions, all other reporting guidelines for systematic reviews indexed in the EQUATOR Network library in March 2018, and other documents, such as conduct guidance and appraisal tools for systematic reviews. One author (MJP) extracted all reporting guidance from the PRISMA 2009 statement and any additional reporting guidance for systematic reviews available in the 60 documents. The same author also reviewed 43 published comments on the PRISMA 2009 statement (identified in an earlier scoping review (13)) and extracted any suggested revisions to items not already captured from the 60 documents.

\subsection{Step 2. Survey of systematic review methodologists and editors}

\subsubsection{Procedure}

We undertook a survey of systematic review methodologists and journal editors to gather their feedback on suggested modifications to PRISMA 2009 items and on the additional items collated, and to identify gaps where new items might be required. Individuals were invited via email to complete the survey, which was created using the online software Qualtrics (Qualtrics, Provo, UT, USA). The survey was open between 6 June 2018 and 31 July 2018, with one reminder email sent on 25 June 2018 to individuals who had not completed the survey and had not indicated that they wished to opt out.

\subsubsection{Ethics}

Ethics approval for the survey was obtained from the Monash University Human Research Ethics Committee (approval number 12813). We informed participants that their responses would be de-identified and that findings would be reported in aggregate. 


\subsubsection{Participants}

We constructed a sampling frame, consisting of all members of the PRISMA 2009 and PRISMA for Protocols (PRISMA-P) 2015 Groups (25, 26), corresponding authors of all PRISMA extensions that were published (27-34) or in development at the time of the survey (35-38), editors in chief and associate editors for two journals specialising in systematic review methodology (BMC Systematic Reviews and Research Synthesis Methods), convenors of the 17 Cochrane Methods Groups (convenors are experienced researchers who are involved in research, development or evaluation in specific areas of systematic review methodology), and all members of the Cochrane Scientific Committee (who are responsible for making decisions about the implementation of new methods for Cochrane reviews). Inclusion of methodologists that contribute to Cochrane provided broad representation because these methodologists are employed across many institutions, and as such, undertake reviews outside of Cochrane, including reviews for guidelines, national government and international organisations (e.g. WHO). The compiled list of individuals was sent to the core team for their review, where they were provided with the opportunity to nominate other individuals. The survey was sent to all individuals on the final list, which consisted of 220 individuals.

\subsubsection{Survey content}

The survey included three parts (see supplement 2 in the Appendix). In Part A, we sought respondents' views on each of the 27 items in the PRISMA 2009 statement, to determine whether they thought the item was still required, and if so, whether it needed modifying. For each item, we presented its current wording in the checklist, along with the explanation and elaboration. In addition, we presented a consideration to prompt thought about whether and 
why the item might need modifying. For example, checklist item 22 in the PRISMA 2009 statement recommends authors "Present results of any assessment of risk of bias across studies." In the consideration for the item, we stated, "The phrase "risk of bias across studies" is ambiguous; it is meant to refer only to reporting biases (e.g., publication bias, selective reporting within studies), but some users may understand it to mean bias in a synthesis resulting from inclusion of studies at risk of bias due to other methodological flaws (e.g. lack of allocation concealment)."

The consideration for each item was based on information collated in the review of existing reporting guidance for systematic reviews (24). For each checklist item, we asked respondents whether we should:

a) keep the checklist item as it is;

b) modify the checklist item using wording suggested by the core team;

c) modify the checklist item otherwise (if selected, respondents were prompted to explain how to modify the item);

d) remove the checklist item.

Respondents were also given an option to select "No opinion". Similar responses options were provided for the question about the current explanation and elaboration for each item. A free text box was provided for respondents to justify their response to each question. The survey did not seek feedback on the PRISMA 2009 flow diagram.

In Part B, we asked respondents whether they believed the updated PRISMA statement should recommend reporting of 12 new items that we identified from the review of existing reporting guidance for systematic reviews (24), and invited them to justify their response or suggest alternative wording for each new item. In Part C, we sought respondents' views on 
the format of the checklist (i.e. single sentence per item, list of sub-items for some or all items, or another format). We also encouraged them to suggest additional reporting items not mentioned in the survey that they believed should be included in the updated guideline.

\subsubsection{Analysis}

We calculated frequencies and percentages of each response option for each question, based on all the available data for that question (i.e. responses from those who partially completed the survey were retained). For an item to reach consensus, one of the response options to the question needed to be rated by more than $66 \%$ of survey respondents; this threshold was selected based on what had been used in previous studies to develop reporting guidelines (33, 39). Items for which there was no consensus, or for which more than $66 \%$ of survey responses selected "remove the checklist item", were considered high-priority items for discussion at the in-person meeting. Responses to the open-ended questions were read by one author (MJP), who summarised the comments, keeping all unique ideas (regardless of the frequency with which they were made). Representative quotes were also included in the summary.

\subsection{Step 3. PRISMA update meeting}

A two-day in-person meeting was held in September 2018 in Edinburgh, Scotland, to discuss the draft content and wording of the updated PRISMA statement, as informed by the review and survey results. Prior to the meeting, participants were emailed a report summarising the results of the survey and a list of all de-identified comments from survey respondents. There were 21 attendees from seven countries, consisting of members of the core team and others selected because of their:

(i) experience in authoring, peer reviewing or editing systematic reviews; 
(ii) expertise in at least one of the following methodological areas: registration and protocols for systematic reviews, question formulation for systematic reviews, searching for studies, study selection, data collection, risk of bias assessment, certainty assessment, meta-analysis, alternative statistical synthesis methods, automation of systematic review processes, and research/knowledge translation; or

(iii) experience producing systematic reviews with or for health care providers, policy makers, regulatory bodies, or other users, and knowledge about what content is required in systematic review reports to meet such user's needs.

All meeting attendees agreed to join the authorship team for PRISMA 2020. Members of the authorship team (all core team members, meeting attendees and four others who were unable to attend the meeting) had a broad range of experience encompassing the development of clinical practice guidelines; working in clinical practice; serving on advisory committees for medical societies, funders, health insurance agencies, and regulatory agencies; working with developers of software for systematic reviews; and, consulting with policy makers. The team had authored reviews independently and under the auspices of evidence synthesis organisations such as Agency for Healthcare Research and Quality (AHRQ), Cochrane and The Campbell Collaboration.

In the meeting we discussed proposed modifications to the PRISMA 2009 checklist items and explanation and elaboration, and the addition of new items. We discussed how to deal with any PRISMA 2009 items identified as potentially problematic because of infrequent reporting (see section 2.1), considering the views about the item gathered in the survey, and reflecting on possible reasons for poor adherence (e.g. journal word/table/figure limits). We also 
discussed modifications to the PRISMA 2009 flow diagram as suggested by other researchers (40-43), which were identified via the review of documents providing reporting guidance for systematic reviews (24), described in section 2.1. We concluded the meeting with a discussion about dissemination and implementation plans. At the start of each day, we asked attendees to consider the following guiding principles when considering content for inclusion in the updated PRISMA statement:

a) the guideline should provide guidance on reporting of systematic reviews only and is not intended to convey guidance on how to conduct systematic reviews;

b) checklist items should specify the minimum that we recommend authors report in all systematic reviews and meta-analyses;

c) authors' adherence to all checklist items should help users assess the risk of bias or applicability of the systematic review findings, or facilitate replication of the systematic review, that is, enable others to repeat the methods used by the reviewers as closely as possible; and

d) the explanation and elaboration can include additional recommendations for reporting and so should be read in conjunction with the checklist.

Discussion of each checklist item proceeded in a standardised format, supported by relevant material presented on slides. A speaker (MJP or JEM) first presented the PRISMA 2009 checklist item and its explanation and elaboration, followed by the suggested modification to each that was presented in the survey, and a summary of the quantitative and qualitative survey data for the item. The speaker then presented the lead author's proposal for how to word the checklist item and its explanation and elaboration in the updated guideline, which was based on data gathered in the survey. A chairperson prompted attendees to share their views on the proposal and to suggest alternative content or wording. At the end of each 
discussion session, the chairperson summarised the main points and noted whether there was support for the proposal presented or that further work on the item was required. No voting on the content or wording of items was conducted. A notetaker (MJP or JEM) recorded the main discussion points, suggestions and decisions made about items.

\subsection{Step 4. Post-meeting activities}

Following the meeting, the lead author of PRISMA 2020 (MJP) prepared a working document including a first draft of the updated PRISMA checklist along with notes about what to include in the explanation and elaboration for each item and a draft of the updated flow diagram, which was sent to the core team members for review. Two members (JEM and DM) discussed with the lead author the feedback received and reviewed a second draft of the working document. Two more drafts were subsequently sent throughout 2019 to the authorship team.

A presentation summarising the development process and main changes made was prepared for the 2019 Cochrane Colloquium and uploaded to YouTube in November 2019 (44). The video concluded with an invitation for interested participants to provide feedback on a preliminary version of the PRISMA 2020 checklist.

In December 2019, members of the authorship team were assigned one or more items to draft the explanation and elaboration and to find an example that best illustrated reporting of that item(s). The drafts were collated and edited by the lead author, and a paper including the checklist, explanation and elaboration and examples for all items was circulated to all coauthors for review. While members of the authorship team were reviewing the paper, a preliminary version of the updated PRISMA checklist was sent in April 2020 to a 
convenience sample of 22 systematic reviewers who had expressed an interest in providing feedback on the checklist (responding to the request for feedback in the November 2019 presentation). Their feedback was considered by the lead author and revisions were incorporated into the second draft of the paper. Comments on the second draft were sought from all members of the authorship team, who then endorsed the final version of the guideline by indicating via email that they had no further comments and approved submission of the guideline for publication.

\section{RESULTS}

\subsection{Step 1. Review of literature to inform the guideline}

Collectively, the four previous studies evaluating the reporting of systematic reviews revealed several PRISMA 2009 items not reported in at least a third of the reviews sampled (13, 2123). These items included systematic review registration details or existence of a systematic review protocol, a full electronic search strategy for databases and study registers searched, methods and results of risk of bias assessments, methods used to explore heterogeneity of results or robustness of syntheses, and funding source for the systematic review.

The review of existing reporting guidance for systematic reviews (24) resulted in the generation of a bank of 221 unique reporting items for systematic reviews, and revealed that all topics addressed by the PRISMA 2009 statement could be modified or supplemented with additional guidance (item bank available at https://osf.io/kbj6v/). The topics addressed by the PRISMA 2009 statement with the highest number of additional items compiled were information sources used to identify studies, data items collected from studies, methods for synthesizing results, study characteristics presented, and results of syntheses (24). 


\subsection{Step 2. Survey of systematic review methodologists and editors}

Among the 220 people invited to participate, 108 fully and two partially completed the survey. The 110 respondents (53 women) were based in 22 countries (17 high income); 74\% of respondents resided in Australia, Canada, the United Kingdom or the United States; and 27\% lived in a country where English was not the primary language spoken. Furthermore, 38\% of survey respondents had co-authored at least one Cochrane review throughout their career.

More than $66 \%$ of respondents recommended keeping six of the PRISMA 2009 checklist items as they were and modifying 15 of the checklist items using the wording suggested by the core team (supplement 3 in the Appendix). The consensus threshold of $66 \%$ was not met for the following six items: abstract, objectives, eligibility criteria, information sources, data items and limitations. The percentage of participants suggesting we remove a PRISMA 2009 item from the updated checklist never exceeded $4 \%$. More than $66 \%$ of respondents supported the inclusion of five of 12 potential new items (supplement 4 in the Appendix). Also, $72 \%$ of respondents preferred changing the format of the checklist to a list of sub-items. Furthermore, $18 \%$ of participants suggested additional items for consideration. Many respondents provided detailed comments to justify their responses; a summary of the comments is provided in supplement 5 in the Appendix.

\subsection{Step 3. PRISMA update meeting}

Discussions at the September 2018 meeting led to broad agreement about the content and wording for some items, while suggestions for further consideration were generated for the majority of items (a list of the proposals presented at the meeting, summary of the main discussion points and decisions made is provided in supplement 6 in the Appendix). Briefly, the following key decisions were made at the meeting: 
- retain all 27 items in the PRISMA 2009 statement using current or modified wording;

- replace the item on the abstract with an updated PRISMA for Abstracts checklist;

- split items which comprise multiple elements into sub-items to facilitate clarity;

- consider creating sub-items recommending authors report: (i) any amendments from the systematic review registration or protocol; (ii) how review outcomes were defined; (iii) methods required to handle data prior to synthesis; (iv) data synthesis methods (meta-analysis and other); and (v) methods used to explore heterogeneity or assess robustness of syntheses and their corresponding results;

- consider including new items recommending authors report (i) how certainty or confidence in the body of evidence was assessed and the results of such assessments; and (ii) whether data, analytic code and other materials used in the review were publicly accessible.

In addition to these decisions, there was general agreement that the PRISMA statement should provide reporting guidance relevant to automation, given many tools to automate or semi-automate several review processes exist or are being developed. Attendees also proposed that consistent wording be used throughout the guideline to describe processes that apply to multiple steps of the review, such as involvement of multiple reviewers to minimise errors (e.g. in study selection, data collection and risk of bias assessment).

\subsection{Step 4. Post-meeting activities}

All the key decisions from the meeting were incorporated into the first draft of the PRISMA 2020 working document. The content and wording of the PRISMA 2020 statement (including the checklists, explanation and elaboration and flow diagrams) were then refined iteratively in response to feedback on drafts of the working document from members of the authorship team. Fifteen systematic reviewers who reviewed a near-final draft of the checklist provided 
feedback which led to minor changes to checklist items and the explanation and elaboration to enhance clarity, but no items were added or removed.

\subsection{Differences between the PRISMA 2009 and 2020 statement}

The PRISMA 2020 statement (45) differs from the PRISMA 2009 statement in several ways (a list of changes to each item with rationale is provided in supplement 7 and the alignment of the checklist items between statements is presented in supplement 8 in the Appendix). The wording of all checklist items was modified to accommodate reporting guidance for new/updated methods; enhance clarity for authors; facilitate replicability of reviews; facilitate assessments of the validity and applicability of reviews; ensure the item is applicable to a wider population of reviews; remove redundancy across items; or for another reason. We created a new section at the end of the checklist pertaining to administrative information, such as funding, which mirrors the style used in other reporting guidelines $(15,17,46,47)$. Otherwise, the same broad sections in PRISMA 2009 appear in PRISMA 2020.

PRISMA 2020 seeks the reporting of the following additional information compared with PRISMA 2009:

- how studies were grouped for the syntheses;

- full search strategies for all databases, registers and websites searched, not just at least one database;

- if applicable, details of automation tools used at various stages of the review (e.g. study selection process, data collection process, risk of bias assessment process);

- a list and definition of all outcomes for which data were sought and methods used to decide which results to collect from included studies;

- the processes used to decide which studies were eligible for each synthesis; 
- any methods required to tabulate or visually display results of individual studies and syntheses;

- any alternative statistical synthesis method used when it was not possible to conduct a meta-analysis;

- any methods used to assess certainty (or confidence) in the body of evidence for an outcome, and assessments for each outcome assessed;

- citations of studies that might appear to meet the inclusion criteria, but which were excluded, and an explanation of why they were excluded;

- the characteristics and risk of bias among studies contributing to each synthesis;

- any amendments to information provided at registration or in the protocol for the review;

- any competing interests of review authors, and

- an indication of whether data, analytic code and other materials used in the review are publicly available and if so, where they can be found.

We also revised the PRISMA flow diagram template to incorporate updated guidance on how to report the results of the search and selection process, and provide templates specific to original systematic reviews and updated systematic reviews. The explanation and elaboration paper (48) includes bullet points that detail the reporting recommendations for each item, a structure that is new to PRISMA, which was adopted to facilitate implementation of the guidance $(49,50)$.

\section{DISCUSSION}

In this paper we provide detailed documentation of the process we used to update the PRISMA 2009 reporting guideline. We used a four-step process, including reviewing 
relevant literature, conducting a survey of systematic review methodologists and editors, holding a meeting to discuss items, and drafting and refining the guidance. This process meant that the guideline was informed by the literature and opinions of those who have expertise in systematic review methods, journal editors, and systematic reviewers. Documenting the process provides a potential roadmap for others in updating reporting guidelines.

The PRISMA 2020 statement includes several more checklist items than the original PRISMA statement. In our survey of methodologists and editors, some respondents advised against lengthening the checklist due to concerns that doing so could decrease adherence, which is already suboptimal (13), and could make systematic review reports unwieldy. While we recognise that the length of the statement may act as a barrier to some, the addition of new items was deemed necessary to capture advances in systematic review methodology and to enhance the replicability of reviews. Furthermore, as we have emphasized in the statement paper (45) and explanation and elaboration paper (48), we do not expect authors to address each PRISMA 2020 item within the main report of the review; referring to relevant information in publicly accessible protocols or supplementary files may suffice. In future, we plan to develop resources and software to facilitate uptake of PRISMA 2020, such as online tools which prompt complete reporting by authors (49) and detection of incomplete reporting by peer reviewers $(50)$.

There are a range of end users of systematic reviews (e.g. patients, guideline developers, healthcare managers and policy makers) who may seek the reporting, or highlighting, of different information. For example, health care managers and policy makers might want less information on the methods than researchers or guideline developers (51). There may 
therefore be value in undertaking a prioritization exercise with different end users to identify items they consider should be part of a minimum set of reporting items for the main report of a systematic review. Given that different groups of end users will likely prioritize different items, careful consideration would need to be given to the findings of this exercise and their influence on the final product.

Hundreds of reporting guidelines for health research are included in the EQUATOR Network Library, although not all have documented their development methods completely. In an evaluation of 317 published reporting guidelines, Schlüssel found that in many guidelines it was unclear whether the development process included a literature review (36\%), a consensus process (44\%), a Delphi survey (49\%), or piloting or seeking of external feedback on at least one version of the guideline (54\%) (52). Failure to report the development process completely may call into question the credibility of the guideline, given the basis for the selected items is not clear, and consequently lead to less uptake by users. We therefore join other developers (53-55) in encouraging more complete reporting of development methods for new and updated reporting guidelines.

There are several strengths of our development process worth reflecting on. Our comprehensive review of existing reporting guidance for systematic reviews (24) prompted us to consider numerous possible modifications and additions to the PRISMA statement that we are unlikely to have identified via discussion alone. Allowing survey respondents to justify their responses to questions yielded a rich dataset of suggestions for how to improve the PRISMA statement, many of which led to new proposals, which without the survey, may not have been identified. Involving contributors with expertise in different areas ensures that the guideline reflects the latest guidance on various aspects of systematic review 
methodology. Involving contributors with: experience producing systematic reviews with or for key end users (e.g. patients, health care providers, policy makers, regulators); developing clinical practice guidelines; working in clinical practice; serving on advisory committees for various stakeholders; working for developers of software for systematic reviewers; and consulting with policy makers means that the perspectives of end users are considered to some extent.

However, there are also some potential limitations of our process. It is possible that the content of PRISMA 2020 may have differed had we included other end users, such as patients and policy makers, directly in the development process, who may have suggested other items for consideration. Furthermore, it is possible that the discussions at the in-person meeting might have differed had we presented results of a Delphi survey with multiple rounds, rather than of a single survey. However, as acknowledged by Moher et al. in their guidance for developers of reporting guidance, there is no best way to generate the list of items for consideration at an in-person meeting (14), and no empirical evidence to suggest one approach is superior to another in terms of its impact on the final guideline. Also, in lieu of using the Delphi method to reach consensus, all members of the authorship team were given four opportunities to comment on the inclusion and wording of items, which was updated iteratively in response to comments on earlier drafts. The final version was approved by all members of the authorship team, not by a formal vote per se, but by indicating via email that they had no further comments and approved submission of the guideline for publication.

Another potential limitation is that we did not systematically search for empirical studies investigating the consequences of failing to report each item recommended in PRISMA 2020. 
We chose not to do this largely for pragmatic reasons, as we assumed that few such studies would exist, and the ability to identify them is hampered by the lack of standardised terminology used to describe these studies. Instead, our recommendations were informed by the reviews and survey conducted, considerations about which items facilitate replication and help users assess risk of bias and applicability of systematic reviews, and co-authors' experience with authoring and using systematic reviews. Also, external feedback to date has been limited to a small convenience sample of systematic reviewers.

Complete reporting of systematic reviews is essential for users seeking to determine the trustworthiness and applicability of the review findings. We hope that documenting the development process for PRISMA 2020 will provide a useful roadmap for others embarking on similar initiatives and will enhance the acceptance and uptake of the PRISMA 2020 statement.

\section{DECLARATIONS}

\section{Acknowledgements}

We thank the following contributors who co-authored the PRISMA 2020 statement with us: Elie A Akl, Sue E Brennan, Roger Chou, Julie Glanville, Jeremy M Grimshaw, Asbjørn Hróbjartsson, Manoj M Lalu, Tianjing Li, Elizabeth W Loder, Evan Mayo-Wilson, Steve McDonald, Luke A McGuinness, Lesley A Stewart, James Thomas, Andrea C Tricco, Vivian A Welch, and Penny Whiting. We thank the following contributors who completed the survey to inform discussions at the PRISMA 2020 development meeting: Xavier Armoiry, Edoardo Aromataris, Ana Patricia Ayala, Ethan M Balk, Virginia Barbour, Elaine Beller, Jesse A Berlin, Lisa Bero, Zhao-Xiang Bian, Jean Joel Bigna, Ferrán Catalá-López, Anna 
Chaimani, Mike Clarke, Tammy Clifford, Ioana A Cristea, Miranda Cumpston, Sofia Dias, Corinna Dressler, Ivan D Florez, Joel J Gagnier, Chantelle Garritty, Long Ge, Davina Ghersi, Sean Grant, Gordon Guyatt, Neal R Haddaway, Julian PT Higgins, Sally Hopewell, Brian Hutton, Jamie J Kirkham, Jos Kleijnen, Julia Koricheva, Joey SW Kwong, Toby J Lasserson, Julia H Littell, Yoon K Loke, Malcolm R Macleod, Chris G Maher, Ana Marušic, Dimitris Mavridis, Jessie McGowan, Matthew DF McInnes, Philippa Middleton, Karel G Moons, Zachary Munn, Jane Noyes, Barbara Nußbaumer-Streit, Donald L Patrick, Tatiana PereiraCenci, Ba' Pham, Bob Phillips, Dawid Pieper, Michelle Pollock, Daniel S Quintana, Drummond Rennie, Melissa L Rethlefsen, Hannah R Rothstein, Maroeska M Rovers, Rebecca Ryan, Georgia Salanti, Ian J Saldanha, Margaret Sampson, Nancy Santesso, Rafael Sarkis-Onofre, Jelena Savović, Christopher H Schmid, Kenneth F Schulz, Guido Schwarzer, Beverley J Shea, Paul G Shekelle, Farhad Shokraneh, Mark Simmonds, Nicole Skoetz, Sharon E Straus, Anneliese Synnot, Emily E Tanner-Smith, Brett D Thombs, Hilary Thomson, Alexander Tsertsvadze, Peter Tugwell, Tari Turner, Lesley Uttley, Jeffrey C Valentine, Matt Vassar, Areti Angeliki Veroniki, Meera Viswanathan, Cole Wayant, Paul Whaley, and Kehu Yang. We thank the following contributors who provided feedback on a preliminary version of the PRISMA 2020 checklist: Jo Abbott, Fionn Büttner, Patricia Correia-Santos, Victoria Freeman, Emily A Hennessy, Rakibul Islam, Amalia (Emily) Karahalios, Kasper Krommes, Andreas Lundh, Dafne Port Nascimento, Davina Robson, Catherine Schenck-Yglesias, Mary M Scott, Sarah Tanveer and Pavel Zhelnov. We thank Abigail H Goben, Melissa L Rethlefsen, Tanja Rombey, Anna Scott, and Farhad Shokraneh for their helpful comments on the preprints of the PRISMA 2020 papers. We thank Edoardo Aromataris, Stephanie Chang and David Schriger for their helpful peer review comments on the PRISMA 2020 development paper. 


\section{Funding}

There was no direct funding for this research. MJP is supported by an Australian Research Council Discovery Early Career Researcher Award (DE200101618) and was previously supported by an Australian National Health and Medical Research Council (NHMRC) Early Career Fellowship (1088535) during the conduct of this research. JEM is supported by an Australian NHMRC Career Development Fellowship (1143429). TCH is supported by an Australian NHMRC Senior Research Fellowship (1154607). JMT is supported by Evidence Partners Inc. DM is supported in part by a University Research Chair, University of Ottawa. The funders had no role in the study design, data collection and analysis, or preparation of the manuscript.

\section{Competing interests}

All authors have completed the ICMJE uniform disclosure form at www.icmje.org/coi_disclosure.pdf and declare: MJP and DM are editorial board members for the Journal of Clinical Epidemiology; DM is chair of the EQUATOR Network, IB is adjunct director of the French EQUATOR Centre and TCH is co-director of the Australasian EQUATOR Centre, which advocate for the use of reporting guidelines to improve the quality of reporting in research articles. TCH has received personal fees from Elsevier outside the submitted work. JMT received salary from Evidence Partners Inc., creators of DistillerSR software for systematic reviews; Evidence Partners Inc. was not involved in the design or outcomes of the statement and the views expressed solely represent those of the author.

\section{Contributors}

All authors declare to meet the ICMJE conditions for authorship. MJP and DM conceived the plan to update the PRISMA statement. MJP, JEM, PMB, IB, TCH, CDM, LS and DM 
contributed to the design of the literature review and survey. MJP administered the survey and analysed the data. MJP prepared all materials for the development meeting. MJP and JEM presented proposals at the development meeting. MJP and JEM took and consolidated notes from the development meeting. MJP drafted the article. All authors were involved in revising the article critically for important intellectual content. All authors approved the final version of the article. MJP is the guarantor of this work.

\section{Data availability statement}

The individual participant dataset for the survey, including responses to each multiple-choice question, is available at https://osf.io/29ncx/. 


\section{References}

1. Moher D, Liberati A, Tetzlaff J, Altman DG, PRISMA Group. Preferred reporting items for systematic reviews and meta-analyses: the PRISMA statement. BMJ. 2009;339:b2535.

2. Moher D, Liberati A, Tetzlaff J, Altman DG, PRISMA Group. Preferred reporting items for systematic reviews and meta-analyses: the PRISMA statement. J Clin Epidemiol. 2009;62(10):1006-12.

3. Moher D, Liberati A, Tetzlaff J, Altman DG, PRISMA Group. Preferred reporting items for systematic reviews and meta-analyses: the PRISMA statement. Ann Intern Med. 2009;151(4):264-9, W64.

4. Moher D, Liberati A, Tetzlaff J, Altman DG, PRISMA Group. Preferred reporting items for systematic reviews and meta-analyses: the PRISMA statement. PLoS Medicine / Public Library of Science. 2009;6(7):e1000097.

5. Moher D, Liberati A, Tetzlaff J, Altman DG, PRISMA Group. Preferred reporting items for systematic reviews and meta-analyses: the PRISMA statement. International Journal Of Surgery. 2010;8(5):336-41.

6. Moher D, Liberati A, Tetzlaff J, Altman DG. Preferred reporting items for systematic reviews and meta-analyses: the PRISMA Statement. Open Med. 2009;3(3):e123-30.

7. Moher D, Liberati A, Tetzlaff J, Altman DG, PRISMA Group. Reprint--preferred reporting items for systematic reviews and meta-analyses: the PRISMA statement. Phys Ther. 2009;89(9):873-80.

8. Liberati A, Altman DG, Tetzlaff J, Mulrow C, Gotzsche PC, Ioannidis JP, et al. The PRISMA statement for reporting systematic reviews and meta-analyses of studies that evaluate health care interventions: explanation and elaboration. J Clin Epidemiol. 2009;62(10):e1-34. 
9. Liberati A, Altman DG, Tetzlaff J, Mulrow C, Gotzsche PC, Ioannidis JP, et al. The PRISMA statement for reporting systematic reviews and meta-analyses of studies that evaluate healthcare interventions: explanation and elaboration. BMJ. 2009;339:b2700.

10. Liberati A, Altman DG, Tetzlaff J, Mulrow C, Gotzsche PC, Ioannidis JP, et al. The PRISMA statement for reporting systematic reviews and meta-analyses of studies that evaluate health care interventions: explanation and elaboration. Ann Intern Med. 2009;151(4):W65-94.

11. Liberati A, Altman DG, Tetzlaff J, Mulrow C, Gotzsche PC, Ioannidis JP, et al. The PRISMA statement for reporting systematic reviews and meta-analyses of studies that evaluate health care interventions: explanation and elaboration. PLoS Medicine / Public Library of Science. 2009;6(7):e1000100.

12. Liberati A, Altman DG, Tetzlaff J, Mulrow C, Gøtzsche PC, Ioannidis JPA, et al. The PRISMA statement for reporting systematic reviews and meta-analyses of studies that evaluate health care interventions: Explanation and elaboration. Italian Journal of Public Health. 2009;6(4):354-91.

13. Page MJ, Moher D. Evaluations of the uptake and impact of the Preferred Reporting Items for Systematic reviews and Meta-Analyses (PRISMA) Statement and extensions: a scoping review. Systematic reviews. 2017;6(1):263.

14. Moher D, Schulz KF, Simera I, Altman DG. Guidance for developers of health research reporting guidelines. PLoS medicine. 2010;7(2):e1000217.

15. Schulz KF, Altman DG, Moher D. CONSORT 2010 statement: updated guidelines for reporting parallel group randomised trials. BMJ. 2010;340:c332.

16. Campbell MK, Piaggio G, Elbourne DR, Altman DG. Consort 2010 statement: extension to cluster randomised trials. BMJ. 2012;345:e5661. 
17. Bossuyt PM, Reitsma JB, Bruns DE, Gatsonis CA, Glasziou PP, Irwig L, et al. STARD 2015: an updated list of essential items for reporting diagnostic accuracy studies. BMJ. 2015;351:h5527.

18. Boutron I, Altman DG, Moher D, Schulz KF, Ravaud P, for the Consort N. P. T. Group. Consort statement for randomized trials of nonpharmacologic treatments: A 2017 update and a consort extension for nonpharmacologic trial abstracts. Ann Intern Med. 2017.

19. Page MJ. Update of the Preferred Reporting Items for Systematic reviews and MetaAnalyses (PRISMA) statement (registered in the EQUATOR Network library for health research reporting on 13 December 2017) [https://www.equatornetwork.org/library/reporting-guidelines-under-development/reporting-guidelinesunder-development-for-systematic-reviews/\#86].

20. Page MJ, McKenzie JE, Bossuyt PM, Boutron I, Hoffmann T, Mulrow CM, et al. Updating the PRISMA reporting guideline for systematic reviews and meta-analyses. Retrieved from osf.io/2v7mk. DOI: 10.17605/OSF.IO/XFG5N. 2018, February 14.

21. Page MJ, Shamseer L, Altman DG, Tetzlaff J, Sampson M, Tricco AC, et al. Epidemiology and Reporting Characteristics of Systematic Reviews of Biomedical Research: A Cross-Sectional Study. PLoS medicine. 2016;13(5):e1002028.

22. Page MJ, Altman DG, Shamseer L, McKenzie JE, Ahmadzai N, Wolfe D, et al. Reproducible research practices are underused in systematic reviews of biomedical interventions. J Clin Epidemiol. 2018;94:8-18.

23. Page MJ, Altman DG, McKenzie JE, Shamseer L, Ahmadzai N, Wolfe D, et al. Flaws in the application and interpretation of statistical analyses in systematic reviews of therapeutic interventions were common: a cross-sectional analysis. J Clin Epidemiol. 2018;95:7-18. 
24. Page MJ, McKenzie JE, Bossuyt PM, Boutron I, Hoffmann T, Mulrow CD, et al. Mapping of reporting guidance for systematic reviews and meta-analyses generated a comprehensive item bank for future reporting guidelines. J Clin Epidemiol. 2020;118:60-8.

25. Moher D, Shamseer L, Clarke M, Ghersi D, Liberati A, Petticrew M, et al. Preferred reporting items for systematic review and meta-analysis protocols (PRISMA-P) 2015 statement. Systematic reviews. 2015;4:1.

26. Shamseer L, Moher D, Clarke M, Ghersi D, Liberati A, Petticrew M, et al. Preferred reporting items for systematic review and meta-analysis protocols (PRISMA-P) 2015: elaboration and explanation. BMJ. 2015;349:g7647.

27. Welch V, Petticrew M, Tugwell P, Moher D, O'Neill J, Waters E, et al. PRISMAEquity 2012 extension: reporting guidelines for systematic reviews with a focus on health equity. PLoS medicine. 2012;9(10):e1001333.

28. Beller EM, Glasziou PP, Altman DG, Hopewell S, Bastian H, Chalmers I, et al. PRISMA for Abstracts: Reporting Systematic Reviews in Journal and Conference Abstracts. PLoS medicine. 2013;10:e1001419.

29. Hutton B, Salanti G, Caldwell DM, Chaimani A, Schmid CH, Cameron C, et al. The PRISMA extension statement for reporting of systematic reviews incorporating network meta-analyses of health care interventions: checklist and explanations. Ann Intern Med. 2015;162(11):777-84.

30. Stewart LA, Clarke M, Rovers M, Riley RD, Simmonds M, Stewart G, et al. Preferred Reporting Items for Systematic Review and Meta-Analyses of individual participant data: the PRISMA-IPD Statement. JAMA. 2015;313(16):1657-65. 
31. Zorzela L, Loke YK, Ioannidis JP, Golder S, Santaguida P, Altman DG, et al. PRISMA harms checklist: improving harms reporting in systematic reviews. BMJ. 2016;352:i157.

32. Guise JM, Butler ME, Chang C, Viswanathan M, Pigott T, Tugwell P, et al. AHRQ Series on Complex Intervention Systematic Reviews - Paper 6: PRISMA-CI Extension Statement \& Checklist. J Clin Epidemiol. 2017;90:43-50.

33. McInnes MF, Moher D, Thombs BD, McGrath TA, Bossuyt PM, and the PRISMADTA Group. Preferred reporting items for a systematic review and meta-analysis of diagnostic test accuracy studies: The PRISMA-DTA statement. JAMA. 2018;319(4):388-96.

34. Tricco AC, Lillie E, Zarin W, O'Brien KK, Colquhoun H, Levac D, et al. PRISMA extension for scoping reviews (PRISMA-SCR): Checklist and explanation. Ann Intern Med. 2018;169(7):467-73.

35. Kapadia MZ, Askie L, Hartling L, Contopoulos-Ioannidis D, Bhutta ZA, Soll R, et al. PRISMA-Children (C) and PRISMA-Protocol for Children (P-C) Extensions: a study protocol for the development of guidelines for the conduct and reporting of systematic reviews and meta-analyses of newborn and child health research. BMJ Open. 2016;6(4):e010270.

36. Rethlefsen M, Koffel J, Kirtley S. PRISMA-Search: guidelines for reporting systematic review literature searches (registered 17 February 2016) http://www.equator-network.org/library/reporting-guidelines-under-development/\#57 [accessed 16 August 2017].

37. Bian Z. Preferred Reporting Items for Systematic Review and Meta-Analyses of traditional Chinese medicine: the PRISMA-TCM Statement (registered 18 August 
2016) http://www.equator-network.org/library/reporting-guidelines-underdevelopment/\#65 [accessed 16 August 2017].

38. Stevens A. PRISMA-RR 2017: an extension to PRISMA for rapid reviews (registered 4 November 2015) http://www.equator-network.org/library/reporting-guidelinesunder-development/\#51 [accessed 16 August 2018].

39. Cohen JF, Korevaar DA, Gatsonis CA, Glasziou PP, Hooft L, Moher D, et al. STARD for Abstracts: essential items for reporting diagnostic accuracy studies in journal or conference abstracts. BMJ. 2017;358:j3751.

40. Mayo-Wilson E, Li T, Fusco N, Dickersin K. Practical guidance for using multiple data sources in systematic reviews and meta-analyses (with examples from the MUDS study). Research synthesis methods. 2018;9(1):2-12.

41. Stovold E, Beecher D, Foxlee R, Noel-Storr A. Study flow diagrams in Cochrane systematic review updates: an adapted PRISMA flow diagram. Systematic reviews. 2014;3:54.

42. Haddaway NR, Macura B, Whaley P, Pullin AS. ROSES RepOrting standards for Systematic Evidence Syntheses: pro forma, flow-diagram and descriptive summary of the plan and conduct of environmental systematic reviews and systematic maps. Environmental Evidence. 2018;7:7.

43. Boers M. Graphics and statistics for cardiology: designing effective tables for presentation and publication. Heart. 2018;104(3):192-200.

44. Page MJ, McKenzie JE, Bossuyt P, Boutron I, Hoffmann T, Mulrow CD, et al. Preferred Reporting Items for Systematic reviews and Meta-Analyses (PRISMA) 2019 Statement: updated guidelines for reporting systematic reviews and metaanalyses. In: Abstracts of the 26th Cochrane Colloquium, Santiago, Chile. Cochrane Database of Systematic Reviews. 2019(1 Suppl 1):102-3. 
https://doi.org/10.1002/14651858.CD201901. Video available at https://www.youtube.com/watch?v=Y-fu00PSm9o.

45. Page MJ, McKenzie JE, Bossuyt PM, Boutron I, Hoffmann TC, Mulrow CD, et al. The PRISMA 2020 statement: an updated guideline for reporting systematic reviews. MetaArXiv. 2020, September 14. DOI: 10.31222/osf.io/v7gm2.

46. von Elm E, Altman DG, Egger M, Pocock SJ, Gotzsche PC, Vandenbroucke JP. Strengthening the Reporting of Observational Studies in Epidemiology (STROBE) statement: guidelines for reporting observational studies. BMJ. 2007;335(7624):806-8.

47. Collins GS, Reitsma JB, Altman DG, Moons KG. Transparent Reporting of a multivariable prediction model for Individual Prognosis or Diagnosis (TRIPOD): the TRIPOD statement. Ann Intern Med. 2015;162(1):55-63.

48. Page MJ, Moher D, Bossuyt PM, Boutron I, Hoffmann TC, Mulrow CD, et al. PRISMA 2020 explanation and elaboration: updated guidance and exemplars for reporting systematic reviews. MetaArXiv. 2020, September 14. DOI: 10.31222/osf.io/gwdhk.

49. Barnes C, Boutron I, Giraudeau B, Porcher R, Altman DG, Ravaud P. Impact of an online writing aid tool for writing a randomized trial report: the COBWEB (Consortbased WEB tool) randomized controlled trial. BMC medicine. 2015;13:221.

50. Chauvin A, Ravaud P, Moher D, Schriger D, Hopewell S, Shanahan D, et al. Accuracy in detecting inadequate research reporting by early career peer reviewers using an online CONSORT-based peer-review tool (COBPeer) versus the usual peerreview process: a cross-sectional diagnostic study. BMC medicine. 2019;17(1):205.

51. Marquez C, Johnson AM, Jassemi S, Park J, Moore JE, Blaine C, et al. Enhancing the uptake of systematic reviews of effects: what is the best format for health care 
managers and policy-makers? A mixed-methods study. Implementation science : IS. 2018;13(1):84.

52. Schlüssel MM. Strengthening the methodology of reporting guidelines: mapping the landscape, updating development guidance and creating a methodological quality badging system. https://osf.io/ebguf. 2020.

53. Korevaar DA, Cohen JF, Reitsma JB, Bruns DE, Gatsonis CA, Glasziou PP, et al. Updating standards for reporting diagnostic accuracy: the development of STARD 2015. Research integrity and peer review. 2016;1(1):7.

54. Dimairo M, Coates E, Pallmann P, Todd S, Julious SA, Jaki T, et al. Development process of a consensus-driven CONSORT extension for randomised trials using an adaptive design. BMC medicine. 2018;16(1):210.

55. Sones W, Julious SA, Rothwell JC, Ramsay CR, Hampson LV, Emsley R, et al. Choosing the target difference and undertaking and reporting the sample size calculation for a randomised controlled trial - the development of the DELTA(2) guidance. Trials. 2018;19(1):542. 


\section{Author statement}

All authors declare to meet the ICMJE conditions for authorship. MJP and DM conceived the plan to update the PRISMA statement. MJP, JEM, PMB, IB, TCH, CDM, LS and DM contributed to the design of the literature review and survey. MJP administered the survey and analysed the data. MJP prepared all materials for the development meeting. MJP and JEM presented proposals at the development meeting. MJP and JEM took and consolidated notes from the development meeting. MJP drafted the article. All authors were involved in revising the article critically for important intellectual content. All authors approved the final version of the article. MJP is the guarantor of this work. 


\section{Competing interests}

All authors have completed the ICMJE uniform disclosure form at www.icmje.org/coi_disclosure.pdf and declare: MJP and DM are editorial board members for the Journal of Clinical Epidemiology; DM is chair of the EQUATOR Network, IB is adjunct director of the French EQUATOR Centre and TCH is co-director of the Australasian EQUATOR Centre, which advocate for the use of reporting guidelines to improve the quality of reporting in research articles. TCH has received personal fees from Elsevier outside the submitted work. JMT received salary from Evidence Partners Inc., creators of DistillerSR software for systematic reviews; Evidence Partners Inc. was not involved in the design or outcomes of the statement and the views expressed solely represent those of the author. 